\title{
Historical control clinical trial to assess the effectiveness of teflubenzuron for treating sea lice on Atlantic salmon
}

\author{
P. J. Campbell ${ }^{1,3}$, K. L. Hammell ${ }^{1, *}$, I. R. Dohoo ${ }^{1}$, G. Ritchie ${ }^{2}$ \\ ${ }^{1}$ Department of Health Management, Atlantic Veterinary College, 550 University Avenue, Charlottetown, \\ Prince Edward Island C1A 4P3, Canada \\ ${ }^{2}$ Nutreco Aquaculture Research Centre, PO Box 48, Sjohagen 3, 4001 Stavanger, Norway \\ ${ }^{3}$ Present address: Inwood Animal Center, 7611 Winchester Avenue, Inwood, West Virginia 25428, USA
}

\begin{abstract}
A historical control clinical trial was performed to assess the effectiveness of teflubenzuron in controlling sea lice Lepeoptheirus salmonis burdens on farmed Atlantic salmon Salmo salar over time. The study site comprised 9 sea cages, all of which were treated. The teflubenzuron was administered in the feed, at a dosage of $10 \mathrm{mg} \mathrm{kg}^{-1}$ biomass $\mathrm{d}^{-1}$, over a treatment period of $7 \mathrm{~d}$. At 1 wk post-treatment, sea lice chalimus and mobile stages were reduced by 92 and $74 \%$ (both $\mathrm{p}<$ 0.001 ), respectively. At 2 wk post-treatment, chalimus stages were reduced by $41 \%$ and mobile stages $61 \%$ (both $\mathrm{p}<0.001$ ) compared to pre-treatment levels. At 3 wk post-treatment, chalimus stages were still $36 \%(p<0.001)$ lower than pre-treatment levels, but mobile stages had increased to above pre-treatment levels. Our results show that the effects of teflubenzuron are limited to a $3 \mathrm{wk}$ duration, but that with appropriate management, farms could benefit from these reduced lice burdens for longer periods.
\end{abstract}

KEY WORDS: Sea lice - Lepeoptheirus salmonis - Teflubenzuron - Atlantic salmon · Salmo salar · Clinical trial

\section{INTRODUCTION}

Teflubenzuron is an orally administered chitin synthesis inhibitor. It has been shown to be effective against all stages of sea lice that undergo a molt, including the larval and pre-adult stages (Ritchie 1996, Branson et al. 2000, Ritchie et al. 2002). It is likely to be used as a sea lice treatment for all cages at a site simultaneously. In a randomized clinical trial reported in Campbell et al. (2006, this issue), half of the cages at the site were medicated and half were negative controls. It has been demonstrated that mobile stages of Lepeoptheirus salmonis can transfer between fish, and between sea cages within a site (Ritchie 1997). Consequently, it is possible that untreated cages in the randomized clinical trial pro- vided a reservoir of lice, some of which may have moved to the treated cages, thus reducing the apparent treatment effect.

The objective of this study was to monitor the effectiveness of teflubenzuron after a $7 \mathrm{~d}$ treatment period under conditions of routine use and exposure rates. All cages were treated in order to assess the impact of treatment under conditions similar to the intended use of the product. Post-treatment lice counts were compared to pre-treatment lice counts. Although the use of historical controls is not usually a preferred method in clinical trials, this design allowed for assessment with natural recruitment of lice from the environment without the potential interference caused by recruitment from non-treated cages at the same site. 


\section{MATERIALS AND METHODS}

Trial design. This was a historical control clinical trial involving all cages at 1 Atlantic salmon Salmo salar cage site. The study began on July 8 and ended on August 8, 1996. Pre-treatment samples were taken from all cages to determine lice infestation levels and to obtain the mean fish weight for biomass estimates. This was followed by a $7 \mathrm{~d}$ treatment with teflubenzuron. Post-treatment samples were taken 7,14 , and $23 \mathrm{~d}$ after the end of treatment. Post-treatment lice counts were compared to pre-treatment lice counts to assess the effectiveness of the teflubenzuron.

Site description. The study site was located near Deer Island, New Brunswick, Canada. There were 9 cages at the site; all were circular $(70 \mathrm{~m}$ circumference). Six of the cages held pre-market fish (1995 year class) and 3 held smolts (1996 year class). The cages with pre-market fish contained 18000 to 20000 fish each, and the smolt cages contained 35000 to 40000 fish each.

Treatment. Teflubenzuron was administered at a dosage of $10 \mathrm{mg} \mathrm{kg}^{-1}$ biomass d $\mathrm{d}^{-1}$ as a medicated feed (dose and duration based on challenge studies by our sponsor, Moore-Clark Canada Co.). The concentration of the teflubenzuron in the medicated feed was $2.0 \mathrm{~kg}$ $\mathrm{t}^{-1}$. The feeding rate for the medicated feed was set at a rate of $0.5 \%$ biomass $\mathrm{d}^{-1}$. The fish received the treatment for a period of $7 \mathrm{~d}$ consecutively.

The medicated feed was delivered in plastic bags which were labelled with site and cage identification, weight of feed in the bag, and the number of bags that each cage was to receive daily. Each cage had a label with the same information.

The fish were fed to satiation twice a day. If the predetermined amount of medicated feed was insufficient to satiate the fish within a cage for the first feeding, non-medicated feed was given as a supplement. If the fish did not eat all of the medicated feed during the first feeding, the remaining medicated feed was given at the second feeding, prior to any non-medicated feed.

Sampling. Sampling was performed by crowding the fish with a seine net, then capturing a small number at a time using a dip net. Sampled fish were anesthetized in a 50 to $100 \mathrm{mg} \mathrm{l}^{-1}$ tricaine methanosulfanate (TMS ${ }^{\mathrm{TM}}$, Syndel International) bath prior to the measurements.

The following measurements were made on each fish: (a) fork length, (b) weight, (c) a count of all lice which were categorized as copepodids, chalimus 1 and 2, chalimus 3 and 4, pre-adults, non-gravid adult lice, and gravid females, (d) a subjective score of sea lice damage whereby $0=$ no damage, $1=$ increased mucus, $2=$ small $\left(<2 \mathrm{~cm}^{2}\right)$ areas of superficial dam- age, $3=$ large $\left(>2 \mathrm{~cm}^{2}\right)$ areas of superficial damage, $4=$ small $\left(<2 \mathrm{~cm}^{2}\right)$ areas of deep damage (through the epidermis), and $5=$ large $\left(>2 \mathrm{~cm}^{2}\right)$ areas of deep damage.

Sample size calculations were not performed for the collection of weight sample data. Since dosing calculations were based on the pre-treatment weight samples, 100 fish per cage, the maximum logistically feasible sample size, were sampled during the pretreatment period. Although sample sizes exceeded recommendations published later by Treasurer \& Pope (2000), logistical constraints were the main determinant of sample size. Consequently, 100 fish were anesthetized and weighed. Lice count and damage score data were collected on a random 25 fish sub-sample of these 100 fish, since counters could not logistically accommodate more than 25 individuals at any one sampling time. To improve repeatability, lice counts and damage scores for each sampling time used for analysis in this study were made by one investigator only.

Environmental measurements. Dissolved oxygen and water temperature were measured at a depth between 2.5 and $3 \mathrm{~m}$ each day on which treatment feed was administered, and in each trial cage.

Statistical analysis. All data were entered into a Quattro Pro (Corel) spreadsheet and then transferred to Stata (Stata) statistical software package for analysis.

Average numbers of lice per fish were calculated for each lice stage at each sample period. Cage means were averaged to obtain sample period means and standard deviations.

Significance of lice count differences between pretreatment and each post-treatment sampling period was assessed using random effects linear regression with 'cage' as a random effect, in order to adjust for clustering within cages. Each sampling period was analyzed separately because the effects of the treatment at each specific follow-up period were the outcome of interest as opposed to an overall estimate of an average treatment effect. For the purposes of this analysis, the dependent variable was a log-transformed lice count $[\log (\operatorname{count}+1)]$ to ensure that the data were approximately normally distributed.

The general model for these regression analyses was:

$$
y_{(i, j)}=\alpha+\beta_{1} x_{1}+u_{(j)}+e_{(i, j)}
$$

where $y=\log [($ lice count on $i$ th fish in $j$ th cage $)+1] ; \alpha=$ intercept, $x_{1}=$ dummy variable for treatment; $\beta_{1}=$ coefficients for treatment; $u_{(j)}=$ random effect of the $j$ th cage, and $e_{(i, j)}=$ residual error.

Analyses were performed for: copepodids, chalimus 1 and 2, chalimus 3 and 4, pre-adults, adults, gravids, 


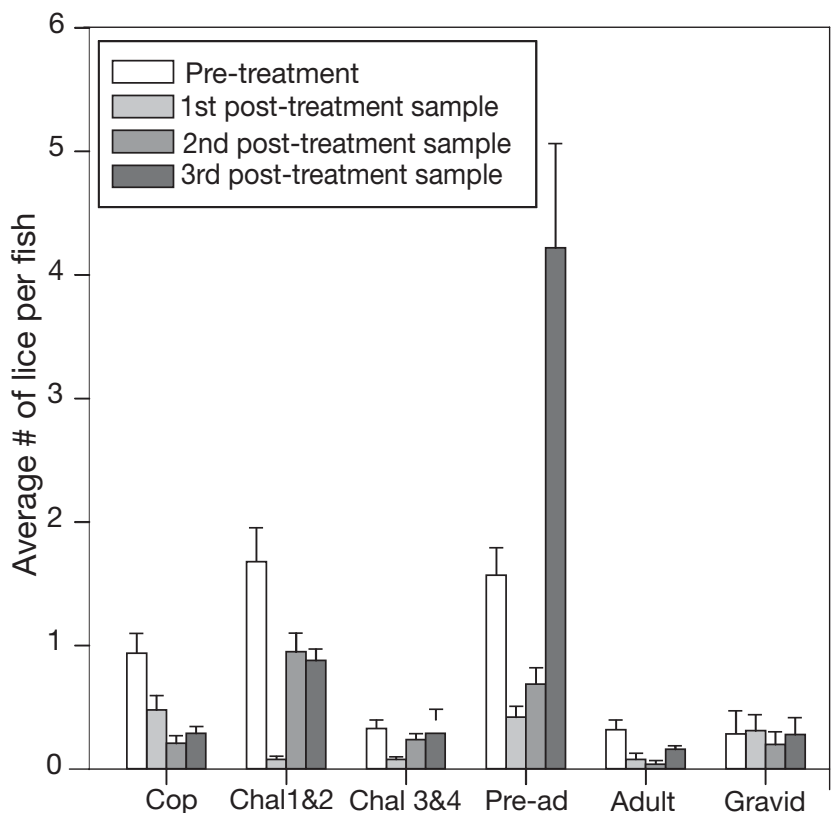

Fig. 1. Lepeoptheirus salmonis on Salmo salar. Average number \pm SEM of lice per fish by stage and sampling period during the historical control clinical trial ( $\mathrm{n}=9$ cages). Cop: copepodid; Chal: chalimus; Pre-ad: pre-adult

all chalimus (1 and 2 plus 3 and 4), mobiles (pre-adult plus adults), and total lice (all stages). Reported mean values are non-adjusted (ie. not log transformed) averages of cage means.

Percent reduction from pre-treatment lice levels (1-[post-treatment mean/pre-treatment mean] $\times 100)$ was calculated and reported where a statistically significant treatment effect $(p<0.05)$ was found.

\section{RESULTS}

\section{Lice counts}

The greatest effect of treatment was seen in the chalimus, pre-adult and adult stages. The average number of lice per fish by stage and sampling period are presented in Fig. 1. At $7 \mathrm{~d}$ after the end of treatment there was a $92 \%$ reduction in chalimus stages and a $74 \%$ reduction in mobile (pre-adult and adult) stages when compared to pre-treatment levels. By $14 \mathrm{~d}$ after the end of treatment the total effect was reduced, although it remained evident, with 41 and $61 \%$ reductions in chalimus and mobiles respectively. All treatment effects appeared to be very much reduced by $23 \mathrm{~d}$ after the end of treatment. Chalimus stages were still $36 \%$ less compared to pre-treatment levels; however, mobile stages had increased to $132 \%$ of pretreatment levels. Copepodids were significantly reduced at all post-treatment sampling periods. Percent reduction in lice numbers by stage and sampling period are presented in Table 1. Reductions were comparable for both year classes in the first 2 time periods, while premarket fish had begun to increase by the third time period (Table 2).

\section{Environmental measurements}

The water temperature during the medication period averaged $11.6^{\circ} \mathrm{C}(\mathrm{SD}=0.7)$, with a minimum of $10.0^{\circ} \mathrm{C}$ and a maximum of $12.7^{\circ} \mathrm{C}$. Dissolved oxygen levels averaged $8.6 \mathrm{mg} \mathrm{l}^{-1}(\mathrm{SD}=0.7)$, with a minimum of $6.8 \mathrm{mg} \mathrm{l}^{-1}$ and a maximum of $11.05 \mathrm{mg} \mathrm{l}^{-1}$ during the $7 \mathrm{~d}$ that experimental feed was administered.

Table 1. Lepeoptheirus salmonis on Salmo salar. Lice count comparisons at each sampling period during the trial (post-treatment sampling periods: $1 \mathrm{st}=7 \mathrm{~d}$; $2 \mathrm{nd}=14 \mathrm{~d}$; $\mathrm{rd}=23 \mathrm{~d}$ ). Data shown are averages of the cage means $(\mathrm{n}=9 \mathrm{cages})$. Values in parentheses are SD. (+): increases rather than reductions in lice numbers. p: results of tests (random effects linear regression) on logtransformed counts. Reduction: \% reduction seen post- as opposed to pre-treatment and was only calculated where a statistically significant difference was detected $(\mathrm{p}<0.05)$. Mobiles: includes pre-adult and adult stages

\begin{tabular}{|c|c|c|c|c|c|c|c|c|c|c|}
\hline & \multirow{2}{*}{$\begin{array}{l}\text { Pre-treatment } \\
\text { Lice/fish }\end{array}$} & \multicolumn{3}{|c|}{ 1st post-treatment } & \multicolumn{3}{|c|}{ 2nd post-treatment } & \multicolumn{3}{|c|}{ 3rd post-treatment } \\
\hline & & Lice/fish & $\mathrm{p}$ & Reduction & Lice/fish & $\mathrm{p}$ & Reduction & Lice/fish & $\mathrm{p}$ & Reduction \\
\hline Copepodids & $0.9(0.5)$ & $0.5(0.3)$ & $<0.001$ & 49 & $0.2(0.2)$ & $<0.001$ & 77 & $0.3(0.1)$ & $<0.001$ & 69 \\
\hline Chalimus 1 \& 2 & $1.7(0.8)$ & $0.1(0.1)$ & $<0.001$ & 95 & $1.0(0.5)$ & $<0.001$ & 44 & $0.9(0.3)$ & $<0.001$ & 48 \\
\hline Chalimus 3 \& 4 & $0.3(0.2)$ & $0.1(0.05)$ & $<0.001$ & 76 & $0.2(0.1)$ & 0.086 & & $0.4(0.2)$ & 0.202 & \\
\hline Pre-adult & $1.6(0.7)$ & $0.4(0.2)$ & $<0.001$ & 73 & $0.7(0.4)$ & $<0.001$ & 56 & $4.2(2.5)$ & $<0.001$ & $(+) 169$ \\
\hline Adult & $0.3(0.2)$ & $0.1(0.1)$ & $<0.001$ & 75 & $0.04(0.1)$ & $<0.001$ & 86 & $0.2(0.1)$ & 0.003 & 50 \\
\hline Gravid & $0.3(0.6)$ & $0.3(0.4)$ & 0.361 & & $0.2(0.3)$ & 0.326 & & $0.3(0.4)$ & 0.272 & \\
\hline All chalimus & $2.0(0.9)$ & $0.2(0.1)$ & $<0.001$ & 92 & $1.2(0.5)$ & $<0.001$ & 41 & $1.3(0.4)$ & $<0.001$ & 36 \\
\hline Mobiles & $1.9(0.7)$ & $0.5(0.4)$ & $<0.001$ & 74 & $0.7(0.4)$ & $<0.001$ & 61 & $4.4(2.5)$ & $<0.001$ & $(+) 132$ \\
\hline Total & $5.1(1.7)$ & $1.4(0.8)$ & $<0.001$ & 72 & $2.3(1.1)$ & $<0.001$ & 54 & $6.2(3.1)$ & 0.026 & $(+) 22$ \\
\hline
\end{tabular}


Table 2. Lepeoptheirus salmonis on Salmo salar. Mean total lice counts (SD) for each year class at each sampling period during the trial

\begin{tabular}{|lcccc|}
\hline & Pre-treatment & 1st post-treatment & 2nd post-treatment & 3rd post-treatment \\
\hline $\begin{array}{c}\text { Smolt } \\
(\mathrm{n}=3 \text { cages })\end{array}$ & $3.5(0.6)$ & $0.7(0.3)$ & $1.2(0.2)$ & $2.6(0.4)$ \\
$\begin{array}{c}\text { Pre-market } \\
(\mathrm{n}=6 \text { cages })\end{array}$ & $5.9(1.4)$ & $1.8(0.7)$ & $2.9(0.8)$ & $8.1(1.7)$ \\
\hline
\end{tabular}

\section{DISCUSSION}

This study was designed as a historical control trial performed concurrently with a randomized clinical trial which used cages as the unit of concern. Although less valuable evidence of treatment effect is gained with a historical control trial, the use of historical controls was warranted in this situation due to the fact that the cost and logistics of randomizing entire sites to no treatment was prohibitive. There were also ethical considerations in having negative control treatment groups in an area with historically high sea lice burdens. If the design had included negative control sites, these sites would have required treatment as soon as 1 cage at the site showed increased lice burdens, since sea lice control is not successful if delays occur. In addition, many sites would have been necessary if 'site' had been used as the level of concern. The primary benefit of carrying out this historical control trial was to evaluate the impact and duration of teflubenzuron in situations of expected use (i.e. entire site treatment).

The effect of treatment was most evident in the stages of lice undergoing a molt. Chalimus stages were reduced by $92 \%$, mobile stages were reduced by $74 \%$, and all stages combined were reduced by $72 \%$ at $1 \mathrm{wk}$ after treatment. Copepodids were significantly reduced at each of the post-treatment sampling periods $(49,77$, and $69 \%$ reductions). Direct effects on this stage would be unexpected because this stage has not yet molted after attaching to the fish. Based on egg incubation time plus longevity of nauplii and copepodid stages (Johnson \& Albright 1991), it is possible that the next generation of sea lice infective copepodids could arise from within-site lice reproduction. The observed reduction in copepodids may be due to adverse effects on the eggs and/or nauplius stages produced by gravid females which were on the medicated fish, resulting in fewer infective copepodids available for recruitment. This supports previous observations that egg strings on gravid females can be damaged after teflubenzuron treatment (Ritchie 1996).

This treatment compares favorably with other treatments available in North America. O'Halloran \&
Hogans (1996) reported that azamethiphos reduced mobile stages by $98 \%$, and chalimus stages by $68 \%$. However, Roth et al. (1996) reported reductions of over $85 \%$ for mobile stages, and no effect on chalimus, in lice susceptible to azamethiphos. Hydrogen peroxide has been reported to remove 44 to $92 \%$ of mobile lice, depending on their stage and sex (Treasurer \& Grant 1997). Emamectin appears to produce similar reductions; however, its effect seems to be considerably more durable (Armstrong et al. 2000, Stone et al. 2000).

The effectiveness of treatment observed in this trial was somewhat higher than reductions seen in the randomized clinical trial (Campbell et al. 2006). This is especially apparent when the differences in methods are considered. This trial measured reductions by comparing post-treatment levels to pre-treatment levels, whereas the randomized trial with untreated control groups measured reductions post-treatment in medicated versus control groups. In a randomized trial, the untreated control groups at sites in this area of the Bay of Fundy would normally be expected to increase in lice numbers as the trial progressed, thereby increasing the difference between control and medicated groups. The results from this historical control trial, in which all cages were treated, suggest that recruitment from untreated cages in the randomized untreated control trial (Campbell et al. 2006) may very well have influenced the observed differences in lice burdens. Alternatively, the observed differences in treatment effect may have been the result of environmental conditions more favorable to lice reproduction and infestation of salmon during the randomized trial which was conducted later in the summer.

Also, in contrast to the historical control trial, there was no effect observed in the copepodids during the randomized clinical trial. It is likely that gravid females in the non-medicated cages in the randomized trial were able to produce a steady supply of normal and infective copepodids.

It was observed that a large number of pre-adult lice suddenly appeared before the third post-treatment sampling period (Table 1). Pre-adults increased from 0.7 per fish at the 2nd post-treatment sampling to 4.2 per fish at the 3rd post-treatment sampling. The 
source of these pre-adults is not clear. There were insufficient larval stages observed in the previous sampling period to account for this increase. The previous sampling period was only $9 \mathrm{~d}$ prior, and it is highly unlikely that this was a sufficient length of time for larval lice to attach as copepodids and grow to the pre-adult stage. According to Johnson \& Albright (1991) it would take approximately $26 \mathrm{~d}$, on average, for copepodids to mature to the first pre-adult stage at $10^{\circ} \mathrm{C}$. Grimnes \& Jakobsen (1996) found a development time of $14 \mathrm{~d}$ from the chalimus 1 and 2 stages to the first pre-adult stage at 9 to $10^{\circ} \mathrm{C}$. Although temperatures were not recorded after treatment, the Department of Fisheries and Oceans monitoring in nearby St. Andrews showed an average temperature of $12.5^{\circ} \mathrm{C}$ for the month of July and $13.1^{\circ} \mathrm{C}$ for August (Ocean Data and Info Services 1999). While these temperatures are admittedly higher than $10^{\circ} \mathrm{C}$, it is unlikely that they were high enough to hasten the development of the lice sufficiently to account for the observed increase. At $10^{\circ} \mathrm{C}$, the chalimus 3 and 4 stages would have time to develop to the pre-adult stages (Johnson \& Albright 1991). Even if the assumption is made that at $13^{\circ} \mathrm{C}$ all of the chalimus stages had sufficient time to develop, an average of less than 1.5 larval lice per fish was observed at the previous sampling, and these, even if they had all developed to adults, still could not account for the observed increase. Despite the unexplained increase in pre-adults, beneficial effects were detected at the third post-treatment sample for 3 of the 6 lice stages.

It is possible that the methods used did not allow for a complete count of all larval stages present. It has been demonstrated that significant proportions of copepodids can be found on the gills and in the buccal cavity (Bron et al. 1991, Johnson \& Albright 1991). If there were chalimus in the gills and buccal cavity, these lice would not have been counted. Subsequently, they would only be detected once they matured to the pre-adult stage and moved out of the gill and buccal cavity. Although this may have acted as a source for the sudden increase in pre-adults, a systemic medication such as teflubenzuron, delivered in the feed and affecting a skin parasite, would also be effective against the same parasite in the highly vascularized tissues of the buccal cavity and gill.

It is also possible that these lice came from outside the site. It has been demonstrated that Lepeophtheirus sp. can transfer between fish in the same cage, and between fish in different cages (Ritchie 1997). It has not been demonstrated how far pre-adults can travel to transfer between hosts. The closest sea-cage site to the study site was approximately $500 \mathrm{~m}$ away and farms with poor lice management would have increasing problems, and therefore influence on the study site, as the summer progressed. Unfortunately, this potential source of lice was impossible to investigate as the lice information from other sites is proprietary.

At the third post-treatment sampling period, lice numbers had recovered to pre-treatment levels. This was $30 \mathrm{~d}$ after the treatment began. This would indicate that treatment with teflubenzuron should be repeated at about $30 \mathrm{~d}$ intervals during the summer, in order to maintain control over the lice populations. Coincidentally, this is the same treatment interval which is recommended when using the chitin synthesis inhibitor lufenuron to control fleas on dogs and cats (Shipstone \& Mason 1995).

Although there are a small number of life stages which did not respond as favorably as expected in this trial, teflubenzuron was generally found to be effective for treating sea lice on farmed salmon. The present study supported previous observations (Wootten et al. 1982, Bron et al. 1993) that treating an entire site at once will probably have a greater impact on a site's overall lice burden than treating cages individually. Trial results also support the suggestion that strategies based on coordinated lice control efforts within a given geographic area may be of greater benefit than individual farm strategies.

Acknowledgements. This study was funded by Moore-Clark Co. (St. Andrews, Canada) and the New Brunswick Salmon Growers Association.

\section{LITERATURE CITED}

Armstrong R, MacPhee D, Katz T, Endris R (2000) A field efficacy evaluation of emamectin benzoate for the control of sea lice on Atlantic salmon. Can Vet J 41:607-612

Branson EJ, Rønsberg SS, Ritchie G (2000) Efficacy of teflubenzuron (Calicide ${ }^{\circledR}$ ) for the treatment of sea lice, Lepeophteirus salmonis (Krøyer 1838), infestations of farmed Atlantic salmon (Salmo salar L.). Aquac Res 31:861-867

Bron JE, Sommerville C, Jones M, Rae GH (1991) The settlement and attachment of early stages of the salmon louse, Lepeophtheirus salmonis (Copepoda: Caligidae) on the salmon host, Salmo salar. J Zool 224:201-212

Bron JE, Sommerville C, Wooten R, Rae GH (1993) Influence of treatment with dichlorvos on the epidemiology of Lepeophtheirus salmonis (Kroyer, 1837) and Caligus elongatus Nordmann, 1832 on Scottish salmon farms. In: Boxshall GA, Defaye D (eds) Pathogens of wild and farmed fish: sea lice. Ellis Horwood, Chichester, p 263-274

Campbell PJ, Hammell KL, Dohoo IR, Ritchie G (2006) Randomized clinical trial to investigate the effectiveness of teflubenzuron for treating sea lice on Atlantic salmon. Dis Aquat Org 70:101-108 (this issue)

Grimnes A, Jakobsen PJ (1996) The physiological effects of salmon lice infection on post-smolt Atlantic salmon. J Fish Biol 48:1179-1194

Johnson SC, Albright LJ (1991) Development, growth, and survival of Lepeophtheirus salmonis (Copepoda: Caligidae) under laboratory conditions. J Mar Biol Assoc UK 71:425-436

Ocean Data and Info Services (1999) Fisheries and Oceans Canada 'Coastal Sea Surface Temperatures-St. Andrews'. 
www.mar.dfo-mpo.gc.ca/science/ocean/coastal_temperature/ Areas/4Xs.html

O'Halloran J, Hogans W (1996) First use in North America of azamethiphos to treat Atlantic salmon for sea lice infestation: procedures and efficacy. Can Vet J 37: 610-611

Ritchie G (1996) Efficacy and action of CME-134 used as an oral treatment for the control of sea lice, Lepeophtheirus salmonis. Bull Aquacult Assoc Can 96(4):26

Ritchie G (1997) The host transfer ability of Lepeophtheirus salmonis (Copepoda: Caligidae) from farmed Atlantic salmon, Salmo salar L. J Fish Dis 20:153-157

Ritchie G, Rønsberg SS, Hoff KA, Branson EJ (2002) Clinical efficacy of teflubenzuron (Calicide ${ }^{\circledR}$ ) for the treatment of Lepeophtheirus salmonis infestations of farmed Atlantic salmon Salmo salar at low water temperatures. Dis Aquat Org 51:101-106

Roth M, Richards RH, Dobson DP, Rae GH (1996) Field trials on the efficacy of the organophosphorus compound azamethiphos for the control of sea lice (Copepoda: Caligidae) infestations of farmed Atlantic salmon (Salmo salar). Aquaculture 140:217-239

Editorial responsibility: Jay F. Levine, Raleigh, North Carolina, USA
Shipstone MA, Mason KV (1995) The use of insect development inhibitors as an oral medication for the control of the fleas Ctenocephalides felis, Ct. canis in the dog and cat. Vet Dermatol 6:131-137

Stone J, Sutherland IH, Sommerville CS, Richards RH, Varma KJ (2000) Field trials to evaluate the efficacy of emamectin benzoate in the control of sea lice, Lepeophtheirus salmonis (Kroyer), infestations in Atlantic salmon, Salmo salar L. Aquaculture 186:205-219

Treasurer JW, Grant A (1997) The efficacy of hydrogen peroxide for the treatment of farmed Atlantic salmon, Salmo salar L. infested with sea lice (Copepoda: Caligidae). Aquaculture 148:265-275

Treasurer JW, Pope JA (2000) Selection of host sample number and design of a monitoring programme for ectoparasitic sea lice (Copepoda: Caligidae) on farmed Atlantic salmon, Salmo salar. Aquaculture 187:247-260

Wootten R, Smith JW, Needham EA (1982) Aspects of the biology of the parasitic copepods Lepeophtheirus salmonis and Caligus elongatus on farmed salmonids, and their treatment. Proc R Soc Edinb Sect B 81:185-197

Submitted: November 1, 2002; Accepted: September 7, 2005 Proofs received from author(s): May 2, 2006 